\title{
From Single Agent to Multi-Agent via $\mathrm{Hy}-$ persequents
}

\author{
Francesca Poggiolesi
}

\begin{abstract}
In this paper we present a sequent calculus for the multiagent system $\mathbf{S} \boldsymbol{5}_{m}$. First, we introduce a particularly simple alternative Kripke semantics for the system $\mathbf{S} \mathbf{5}_{m}$. Then, we construct a hypersequent calculus for $\mathbf{S 5}_{m}$ that reflects at the syntactic level this alternative interpretation. We prove that this hypersequent calculus is theoremwise equivalent to the Hilbert-style system $\mathbf{S} \mathbf{5}_{m}$, that it is contraction-free and cut-free, and finally that it is decidable. All results are proved in a purely syntactic way and the cut-elimination procedure yields an upper bound of $i p_{2}(n, 0)$ where $i p_{2}$ is an hyperexponential function of base 2 .
\end{abstract}

Key words Aumann structures, cut-elimination, decidability, modal logic, multiagent systems

MSC Codes 0B42, 0B45, 0B70, 03F03, 03F05

\section{Introduction}

The starting point of our paper is modal logic, the logic obtained from classical logic by adjoining the symbol $\square$. As is very well-known, the symbol $\square$ has been interpreted in many different ways: the standard interpretation is in terms of necessity, but one can also interpret the box in deontic terms and in an epistemic way. This last interpretation is perhaps that which has received the most attention. The modal system normally chosen for representing knowledge is the system S5. From an epistemic point of view, of greater interest than $\mathbf{S 5}$ is the multi-agent system $\mathbf{S} \boldsymbol{5}_{m}$. Indeed $\mathbf{S} \mathbf{5}_{m}$ is the basic system for obtaining the several systems of dynamic epistemic logic (e.g. see $(3),(5))$ which today represent the most epistemically grounded logics of the market. So $\mathbf{S 5}_{m}$ can be seen as a sort of bridge between modal logic and dynamic epistemic logic.

From a proof-theoretical point of view, the most recent sequent calculus for the system S5 has been proposed by (7). Let us call this calculus HS5. 
HS5 is composed just by the axioms, the propositional rules and three modal rules that introduce the symbol $\square$ on the right and on the left of the sequent (all the structural rules are proved to be admissible). In this respect HS5 is different from many other sequent calculi proposed for the modal logic S5 (e.g. see (4), (6), (11)): in these calculi, beyond the rules mentioned for HS5, there are logical or structural rules that reflect the properties of reflexivity, symmetry and transitivity which are usually required by the accessibility relation of Kripke frames for S5. The reason why HS5 does not need these rules is because it reflects the less complicated semantic interpretation of $\mathbf{S 5}$ where we consider classes of frames which are just non-empty sets of worlds. (Let us call these frames S5 Kripke frames.) As a consequence, HS5 is a simple and elegant sequent calculus for the system $\mathbf{S 5}$.

Given this result, our goal in this paper is to obtain an analogously elegant and simple sequent calculus for $\mathbf{S} \mathbf{5}_{m}$. For this, the calculus will have to reflect a semantic interpretation of the multi-agent setting which is based on sets and not on accessibility relations. As far as we know, there does not exist a Kripke semantic interpretation for $\mathbf{S} \mathbf{5}_{m}$ that is so characterised. On the other hand, there exist Aumann structures (see (1)) which are very close to what we need. By exploiting these structures, we will proceed in the following way: in Section 2 we will introduce an alternative Kripke semantics for the system $\mathbf{S} \boldsymbol{5}_{m}$, and we will show that this semantics is equivalent to the standard one. In Section 3, we will present an indexed hypersequent calculus for $\mathbf{S} \mathbf{5}_{m}$, which reflects the new semantic interpretation presented in Section 2. We will call this calculus $\mathbf{H S 5 _ { m }}$. In Section 4 we will prove that $\mathbf{H S 5}_{m}$ is contraction-free, weakening-free and that its logical rules are invertible, while in Section 5 we will prove that $\mathbf{H S 5}_{m}$ is cut-free. In Section 6 we will show that $\mathbf{H S F}_{m}$ is decidable.

Let us note that the task of providing a sequent calculus for the system $\mathbf{S 5 _ { m }}$ is not only fundamental per se, since, as far as we know, no result has still been obtained in this direction, but, moreover, it represents a first important step towards a proof theory for dynamic epistemic logic which is a field that has been so far largely neglected. (Indeed there are some results in the proof theory for dynamic epistemic logic, e.g. (2), but they mainly concern tableaux systems, and not sequent calculi.)

Let us finally underline the relevance of this paper for the Special Issue Scope of Logic Theorems. The issue mainly deals with the scope of validity for logic and with questions as the following ones: given the increasing number of new logics and the fact that not all of them enjoy the properties of classical logic (e.g. completeness, cut-elimination), what kind of consequences shall we draw? Are they logics or not? In this paper we prove the cut-elimination theorem for a famous non-classical logic, i.e. the multi-agent version of the modal system S5. If one deems as crucial for a logic to enjoy the cut-elimination theorem, then we can claim to have proved that the system $\mathbf{S} \mathbf{5}_{m}$ is a logic in the proper sense of the word. 


\section{An alternative semantic interpretation of $\mathbf{S} \mathbf{5}_{m}$}

The standard Kripke-interpretation of the modal system $\mathbf{S} \mathbf{5}_{m}$ is as follows: $\mathbf{S 5 _ { m }}$ is sound and complete with respect to the class of frames which have $m$ different accessibility relations, all enjoying the three properties of reflexivity, symmetry and transitivity. In other words, $\mathbf{S} \mathbf{5}_{m}$ is sound and complete with respect to the class of frames which have $m$ different equivalent relations. In this section we propose an alternative Kripke semantics interpretation for $\mathbf{S} \mathbf{5}_{m}$ which is based on Aumann structures and where the $m$ accessibility relations do not have to be taken into account. We show that this semantic interpretation of $\mathbf{S} \mathbf{5}_{m}$ is equivalent to the standard one.

We strongly underline that the alternative Kripke semantics of the system $\mathbf{S} \boldsymbol{5}_{m}$ that we present in this section only serves to an intuitively better understanding of the sequent calculus that we will introduce in Section 3. We consider it important to have such an intuitive interpretation. On the other hand, the reader who is not interested in it can easily skip this section.

Definition 2.1. We consider a language $\mathcal{L}_{h}^{\square}$ with a set $\Phi$ of agents $\{a, b, c, \ldots\}$. Propositions $p$ are atoms. The set of atoms is denoted by $\Psi$. Formulas are denoted by capital letters $A, B, C, D \ldots$ They are given by the following grammar:

$$
A::=p|\neg A|(A \wedge A) \mid \square_{z} A
$$

where $z \in \Phi$ and the formula $\square_{z} A$ is read as "agent $z$ knows A".

The other propositional connectives $\vee$ and $\rightarrow$, as well as the modal operator $\diamond_{z}$, are defined as usual.

Definition 2.2. A partition $R_{z}$ of a set $W$ is a set $\left\{W_{1}, \ldots, W_{n}\right\}$ of subsets of $W$ such that the $W_{i}$ 's are disjoint and the union of the $W_{i}$ 's is the set $W$. If $R_{z}=\left\{W_{1}, \ldots, W_{n}\right\}$ is a partition, then the sets $W_{i}$ are called the cells of the partition $R_{z}$, or the information sets of agent $z$.

Definition 2.3. An Aumann-structure $\mathfrak{F}^{A}$ for $h$ agents over $\Phi$ is an h-tuple $\left\langle W, R_{a}, \ldots, R_{h}\right\rangle$, where $W$ is a set of possible worlds, while $R_{z}$ is a partition of $W$, for each agent $z \in \Phi$. We denote with $R_{z}(s)$ the cell of the partition $R_{z}$ where the world $s$ appears.

Aumann structures are like Kripke structures with one main difference: instead of using binary accessibility relations $\mathbb{R}_{a}, \ldots, \mathbb{R}_{h}$ to describe what $h$ different agents belonging to $\Phi$ consider possible, Aumann structures exploit partitions $R_{a}, \ldots, R_{h}$.

Definition 2.4. An Aumann-Kripke model $\mathfrak{M}^{a}$ for $h$ agents over $\Phi$ is a h-tuple $\left\langle W, R_{a}, \ldots, R_{h}, \pi\right\rangle$, where $\left\langle W, R_{a}, \ldots, R_{h}\right\rangle$ is an Aumann-structure and $\pi$ is a function mapping every element in $W \times \Psi$ to a value in $\{0,1\}$. 
Definition 2.5. Given a model $\mathfrak{M}^{a}=\left\langle W, R_{a}, \ldots, R_{h}, \pi\right\rangle$ and $s \in W$, the satisfiability relation $s \models \mathfrak{M}^{a} A$ is defined in the following inductive way:

- $s \models_{\mathfrak{M}^{a}} p$ iff $\pi(s, p)=1$

- $s \models \mathfrak{M}^{a} \neg A$ iff $s \not \nvdash_{\mathfrak{M}^{a}} A$

- $s \models_{\mathfrak{M}^{a}} A \wedge B$ iff $s \models \models_{\mathfrak{M}^{a}} A$ and $s \models_{\mathfrak{M}^{a}} B$

- $s \models_{\mathfrak{M}^{a}} \square_{z} A$ iff $\forall t \in W\left(R_{z}(s)=R_{z}(t) \rightarrow t \models_{\mathfrak{M}^{a}} A\right)$

There is a natural correspondence between partitions on $W$ and equivalence relations on $W$. Given a partition $R_{z}$ on $W$, the corresponding equivalence relation $\mathbb{R}_{z}$ is defined in the following way:

$$
(s, t) \in \mathbb{R}_{z} \text { if, and only if, } R_{z}(s)=R_{z}(t)
$$

In a similar way, given an equivalence relation $\mathbb{R}_{z}$ on $W$, the corresponding partition $R_{z}$ consists of the equivalence classes induced by the equivalence relation.

Lemma 2.6. $\mathbb{R}_{z}$ is the equivalence relation that we obtain from a partition $R_{z}$ if, and only if, $R_{z}$ is the partition that we obtain from the equivalence relation $\mathbb{R}_{z}$.

Proof. Straightforward.

Let $\mathfrak{M}$ be the Kripke model $\left\langle W, \mathbb{R}_{a}, \ldots, \mathbb{R}_{h}, \pi\right\rangle$, where each $\mathbb{R}_{z}$ is an equivalence relation. We define the corresponding Aumann-Kripke model $\mathfrak{M}^{a}$ $=\left\langle W, R_{a}, \ldots, R_{h}, \pi\right\rangle$ in the following way: $\mathfrak{M}$ and $\mathfrak{M}^{a}$ have the same set $W$ and the same evaluation function $\pi$, and each $R_{z}$ is the partition corresponding to the equivalence relation $\mathbb{R}_{z}$.

Lemma 2.7. Let $\mathfrak{M}$ be a Kripke model (with $h$ equivalence relations) and let $\mathfrak{M}^{a}$ the corresponding Aumann-Kripke model. Then, for each formula A, we have: ${ }^{1}$

$$
s \mid=_{\mathfrak{M}^{a}} A \text { if, and only if, }\left.s\right|_{\mathfrak{M}} A
$$

Proof. By induction on A.

Given the Aumann-Kripke model $\mathfrak{M}^{a}=\left\langle W, R_{a}, \ldots, R_{h}, \pi\right\rangle$, then the Kripke model $\mathfrak{M}=\left\langle W, \mathbb{R}_{a}, \ldots, \mathbb{R}_{h}, \pi\right\rangle$ is its correspondent model where each $\mathbb{R}_{z}$ is the equivalence relation corresponding to the partition $R_{z}$. Lemma 2.7 also holds if we first consider Aumann-Kripke models, and then look to the corresponding Kripke models.

We have thus introduced an alternative semantic way to realise the system $\mathbf{S 5}_{m}$. Let us call this semantics $\mathbf{S} 5_{m}$ Aumann-Kripke frames. $\mathbf{S 5}_{m}$ Aumann-Kripke frames should be seen as the multi-agent versions of the $\mathbf{S 5}$

${ }^{1}$ Note that the definition of $\models_{\mathfrak{M}}$ is the standard one. 
Kripke frames. Indeed, in both cases, there is no mention of an accessibility relation; rather, the focus goes on sets. While in the case of S5 Kripke frames we could simply talk about non-empty sets of worlds, in the case of $\mathbf{S 5}_{m}$ Aumann-Kripke frames we have to keep track of the agents occurring in the language, and hence we deal with partitions.

\section{The calculus $\mathbf{H S F}_{m}$}

In this section we introduce the indexed hypersequent calculus $\mathbf{H S 5}_{m}$ which reflects, at the syntactic level, the $\mathbf{S} \mathbf{5}_{m}$ Aumann-Kripke frames that we have illustrated in the previous section.

In what follows we use the following syntactic conventions:

- $M, N, \ldots$ : finite multisets of formulas,

- $\Gamma, \Delta, \ldots$ : classical sequents,

- $G, H, \ldots$ indexed hypersequents,

- $\alpha, \beta, \ldots$ finite sets of indexes of the form $n z$, where $n \in \mathbb{N}$ and $z \in \Phi$, and, for each set $\alpha$ and for each $z \in \Phi$, there exists at most one index $n z \in \alpha$. So, for instance, $\alpha$ could be the set $\{1 a, 1 b, 2 c\}$, but $\{1 a, 2 a\}$ is not a legal set of indexes.

Definition 3.1. An indexed hypersequent is a finite multiset of indexed sequents, i.e. a syntactic object of the form:

$$
\alpha_{1}: M_{1} \Rightarrow N_{1}\left|\alpha_{2}: M_{2} \Rightarrow N_{2}\right| \ldots \mid \alpha_{n}: M_{n} \Rightarrow N_{n}
$$

where $M_{i} \Rightarrow N_{i}(i=1, \ldots, n)$ is a classical sequent, $\alpha_{i}$ is a finite set of indexes as defined above, and, for all $m p, 1 \leq m, p \leq n$ and $m \neq p$,

1. $\alpha_{m} \cap \alpha_{p}$ contains at most one element;

2. there exists a sequence $k_{1}, \ldots, k_{q}$ with $k_{1}=m$ and $k_{q}=p$, and for all $r$, $1 \leq r<q, \alpha_{k_{r}} \cap \alpha_{k_{r+1}} \neq \emptyset$.

3. there does not exist a sequence of indexed sequents $\beta_{1}: P_{1} \Rightarrow Q_{1} \mid \beta_{2}$ : $P_{2} \Rightarrow Q_{2}|\ldots| \beta_{q}: P_{q} \Rightarrow Q_{q}$ such that:

- for each pair of indexed sequents $\beta_{r}: P_{r} \Rightarrow Q_{r}, \beta_{r+1}: P_{r+1} \Rightarrow$ $Q_{r+1}$, with $1 \leq r<q, \beta_{r} \cap \beta_{r+1}$ contains one element;

- $\beta_{1}: P_{1} \Rightarrow Q_{1}$ is the same sequent as $\beta_{q}: P_{q} \Rightarrow Q_{q}$.

Let us call disconnected indexed hypersequent, for short $D I H$, an indexed hypersequent that satisfies 1 and 3, but not necessarily 2 .

Let us intuitively explain what an indexed hypersequent is. The idea is the following. Each sequent is meant to represent a world of an $\mathbf{S} \mathbf{5}_{m}$ AumannKripke frame, while each index $n z$ is meant to indicate a particular cell of a partition. Therefore the set of indexes $\alpha_{i}$ in front of the sequent $M_{i} \Rightarrow$ $N_{i}$ indicates the several different cells (for several different agents) that the world-sequent $M_{i} \Rightarrow N_{i}$ belongs to. 
In order to get a rigourous syntactic interpretation of indexed hypersequents, matters become a little bit more complicated and we need the following several definitions. The notion of of DIH is crucial in these definitions.

Definition 3.2. Let us consider an indexed sequent $\alpha_{i}: \Gamma_{i}$ belonging to an indexed hypersequent $H$. We define the set of all the indexed sequents belonging to $H$ that have at least one common index with $\alpha_{i}: \Gamma_{i}$ in the following way:

$$
\Sigma_{\alpha_{i}: \Gamma_{i}}^{H}=\left\{\alpha_{j}: \Gamma_{j} \mid \alpha_{i} \cap \alpha_{j} \neq \emptyset\right\}
$$

Definition 3.3. We define an indexed hypersequent $H$ from which we drop (i) a sequent $\alpha_{i}: \Gamma_{i}$ and (ii) each of the indexes belonging to $\alpha_{i}$ that occurs in other indexed sequents of $H$, in the following way:

$$
H \backslash \alpha_{i}: \Gamma_{i}=\alpha_{1}^{\prime}: \Gamma_{1}\left|\alpha_{i-1}^{\prime}: \Gamma_{i-1}\right| \alpha_{i+1}^{\prime}: \Gamma_{i+1} \mid \alpha_{n}^{\prime}: \Gamma_{n}
$$

where $\alpha_{j}^{\prime}=\alpha_{j} \backslash \alpha_{i}$. Note that $H \backslash \alpha_{i}: \Gamma_{i}$ is a disconnected indexed hypersequent.

For any $\alpha_{i}, \alpha_{j}$ with a single common element $n z$, we use $f\left(\alpha_{i}, \alpha_{j}\right)$ to denote the agent $\mathrm{z}$. We also use the following abbreviations: $n z: \Gamma$ for $\{n z\}$ : $\Gamma$, and $\Gamma$ for $\emptyset: \Gamma$.

Definition 3.4. The interpretation $\tau$ of a DIH $H$ rooted at $\alpha_{i}: \Gamma_{i},(H)_{\alpha_{i}: \Gamma_{i}}^{\tau}$, is inductively defined in the following way:

- if $H=\Gamma_{i}$ or $H=\Gamma_{i} \mid G$, and $\Gamma_{i}=M \Rightarrow N$, then $(H)_{\alpha_{i}: \Gamma_{i}}^{\tau}=\bigwedge M \rightarrow$ $\bigvee N$

- if $H=\alpha_{1}: \Gamma_{1}|\ldots| \alpha_{i}: \Gamma_{i}|\ldots| \alpha_{n}: \Gamma_{n}$, then $(H)_{\alpha_{i}: \Gamma_{i}}^{\tau}=$

$$
\left(\Gamma_{i}\right)_{\alpha_{i}: \Gamma_{i}}^{\tau} \vee \bigvee_{\alpha_{j}: \Gamma_{j} \in \Sigma_{\alpha_{i}: \Gamma_{i}}^{H}} \square_{f\left(\alpha_{j}, \alpha_{i}\right)}\left(H \backslash \alpha_{i}: \Gamma_{i}\right)_{\alpha_{j}: \Gamma_{j}}^{\tau}
$$

Definition 3.5. The interpretation of an indexed hypersequent $H$ is defined in the following way:

$$
(H)^{\tau}=\bigwedge_{\alpha_{i}: \Gamma_{i} \in H}(H)_{\alpha_{i}: \Gamma_{i}}^{\tau}
$$

We now have almost all the elements to introduce the calculus $\mathbf{H S \mathbf { 5 } _ { m }}$. Let us finish by pointing out the following notations and abbreviations. We use $\alpha^{\wedge} n z$ to denote the set of indices (understood to satisfy the property just mentioned) formed by the union of $\alpha$ and the index $n z$. This notation serves to draw the reader's attention to the index $n z$. We use $\|H\|$ to denote the union of all the sets of indices contained in the hypersequent $H$.

The postulates of the calculus $\mathbf{H S 5 _ { m }}$ are 


\section{Initials Indexed Hypersequents}

$G \mid \alpha: M, p \Rightarrow N, p$

\section{Propositional Rules}

$$
\begin{array}{lc}
\frac{G \mid \alpha: M \Rightarrow N, A}{G \mid \alpha: \neg A, M \Rightarrow N} \neg L & \frac{G \mid \alpha: A, M \Rightarrow N}{G \mid \alpha: M \Rightarrow N, \neg A} \neg R \\
\frac{G \mid \alpha: A, B, M \Rightarrow N}{G \mid \alpha: A \wedge B, M \Rightarrow N} \wedge L & \frac{G|\alpha: M \Rightarrow N, A \quad G| \alpha: M \Rightarrow N, B}{G \mid \alpha: M \Rightarrow N, A \wedge B} \wedge R
\end{array}
$$

\section{Modal Rules}

$$
\begin{aligned}
& \frac{G \mid \alpha: \square_{z} A, A, M \Rightarrow N}{G \mid \alpha: \square_{z} A, M \Rightarrow N} \square L_{1} \\
& \frac{G\left|\alpha \wedge n z: \square_{z} A, M \Rightarrow N\right| \beta^{\wedge} n z: A, P \Rightarrow Q}{G\left|\hat{\alpha} n z: \square_{z} A, M \Rightarrow N\right| \beta^{\wedge} n z: P \Rightarrow Q} \square L_{2}
\end{aligned}
$$$$
\frac{G|\hat{\alpha} n z: M \Rightarrow N| n z: \Rightarrow A}{G \mid \beta: M \Rightarrow N, \square_{z} A} \square R
$$

where in the rule $\square R$, the index $\beta= \begin{cases}\alpha, & \text { if } n z \notin\|G\| \\ \alpha n z, & \text { otherwise }\end{cases}$

\section{Cut Rule}

$$
\frac{G|\alpha: M \Rightarrow N, A \quad G| \alpha: A, M \Rightarrow N}{G \mid \alpha: M \Rightarrow N}{C u t_{A}}
$$

Let us make two remarks on the modal rules. The first one only concerns the rules $\square L_{i}(i=1,2)$. The repetition of the principal formula $\square_{z} A$ in the premise of each of these rules only serves to make the rules invertible. This is analogous to the repetition of the formula $\forall x A(x)$ in the premise of the rule which introduces the symbol $\forall$ on the left side of the sequent in some versions of the sequent calculus for first-order classical logic.

The second remark concerns the three modal rules. It is easy to informally understand these rules if we think of $\mathbf{S} \mathbf{5}_{m}$ Aumann-Kripke frames. Let us first of all consider the two rules $\square L_{i}$. These two rules should be interpreted, if read bottom up and considered together, in the following way. Let us call $s$ the world that corresponds to the sequent $\square_{z} A, M \Rightarrow N$. As we can see from the set of indexes $\alpha \hat{n} z$, the world $s$ belongs to a partition cell for the agent $z$, i.e. the partition cell called $n z$. Hence, if the formula $\square_{z} A$ is 
true at the world $s$ of the partition cell $n z$, then the formula $A$ is true at any world that belongs to the same partition cell $n z$.

Let us now pass to the rule $\square R$. We have two variants of this rule: (i) $\beta=\alpha$, and (ii) $\beta=\hat{\alpha} n z$. Let us first interpret the variant (i) of $\square R$, reading it bottom-up. Let us call $s$ the world that corresponds to the sequent $M \Rightarrow N, \square_{z} A$. Given the form of $\square R$, and the restriction on sets of indexes, we know that the world $s$ does not belong to any partition cell for the agent $z$. Then, if the formula $\square_{z} A$ is false at the world $s$, we can construct a new partition cell for the agent $z$ and a new world $t$, which together with the world $s$ belongs to the partition cell for the agent $z$, such that in the world $t$ the formula $A$ is false.

Let us now pass to the variant (ii) of the rule $\square R$. In this case we know that the world $s$ belongs to a partition cell for the agent $z$, i.e. the partition cell called $n z$. If the formula $\square_{z} A$ is false at the world $s$, then we can construct a new world $t$ that belongs to the partition cell $n z$ where the formula $A$ is false.

\section{Admissibility of the Structural Rules}

In this section we show which structural rules are height and cut-rankpreserving admissible in the calculus $\mathbf{H S} \mathbf{5}_{m}$. Moreover, in order to show that the two rules of contraction are height- and cut-rank-preserving admissible, we show that the propositional and modal rules are height and cut-rankpreserving invertible. The proof of the admissibility of the cut-rule will be given in the sixth section.

Definition 4.1. We associate to each derivation $d$ in $\mathbf{H S 5}_{m}$ two natural numbers $h(d)$ (the height of $d$ ) and $r k(d)$ (cut-rank of $d$ ). The height corresponds to the length of the longest branch in a tree-derivation $d$, where the length of a branch is the number of nodes in the branch minus one; the cut-rank corresponds to the complexity of the cut-formulas in $d . r k(d)$ is the smallest $n \in \mathbb{N}$ such that each cut-formula $A$ occurring in $d$ is such that $d g(A)<n$, where $d g(A)$ stands for the complexity of $A$. If $r k(d)=0$, then $d$ is a cut-free derivation. We do not dwell on the standard inductive definitions of height and cut-rank of a derivation (e.g. see (10)).

Definition 4.2. $\mathbf{H S 5}_{m} \vdash_{p}^{n} G$ means that there exists a derivation $d$ of $G$ in $\mathbf{H S 5}_{m}$, with $h(d) \leq n$ and $r k(d) \leq p$. When needed, we write ${ }_{\langle p\rangle}^{\langle n\rangle} G$ as a shortening of $\mathbf{H S} \boldsymbol{5}_{m} \vdash_{p}^{n} G$.

Definition 4.3. An inference rule $\mathcal{R}$ with premises $G_{1}, \ldots, G_{n}$ and conclusion $H$ is height- and cut-rank-preserving admissible in the calculus $\mathbf{H S}_{m}$ if, whenever $\mathbf{H S 5}_{m} \vdash_{p}^{n} G_{i}$, for each premise $G_{i}$, then $\mathbf{H S 5}_{m} \vdash_{p}^{n} H$. For each rule $\mathcal{R}$, there is its inverse(s), denoted by $\overline{\mathcal{R}}_{i}$ (one for each premise $G_{i}$ of $\mathcal{R}$ ), which has the conclusion of $\mathcal{R}$ as its only premise and any premise of $\mathcal{R}$ as 
its conclusion. An inference rule is height- and cut-rank-preserving invertible in the calculus $\mathbf{H S 5} \mathbf{5}_{m}$ if $\overline{\mathcal{R}}_{i}$ is height- and cut-rank-admissible in $\mathbf{H S} \mathbf{5}_{m}$.

Lemma 4.4. For any indexed hypersequent $G$, if $G$ is derivable in $\mathbf{H S 5}_{m}$, then $G\left[n_{1}^{\prime} z_{1} \ldots n_{k}^{\prime} z_{k} / n_{1} z_{1} \ldots n_{k} z_{k}\right]$ is also derivable with the same height and the same cut-rank, provided that $G\left[n_{1}^{\prime} z_{1} \ldots n_{k}^{\prime} z_{k} / n_{1} z_{1} \ldots n_{k} z_{k}\right]$ is an indexed hypersequent (i.e. that it respects the conditions 1., 2. and 3. of Definition 3.1).

Proof. By straightforward induction on the height of the derivation.

Lemma 4.5. The rule of merge

$$
\frac{G|\hat{\alpha} n z: M \Rightarrow N| n z: P \Rightarrow Q}{G \mid \beta: M, P \Rightarrow N, Q} \text { merge }
$$

where the index $\beta=\left\{\begin{array}{ll}\alpha, & \text { if } n z \notin\|G\| \\ \alpha \hat{n} z, & \text { otherwise, }\end{array}\right.$ is height- and cut-rank preserving admissible in $\mathbf{H S 5}_{m}$.

Proof. By induction on the height of the derivation of the premise. If the premise is an initial indexed hypersequent, then so is the conclusion. If the premise is inferred by a propositional rule or by the rule $\square L_{1}$, then the inference is clearly preserved. If the premise is inferred by the modal rule $\square R$, there are several cases to analyse depending on the index occurring in the indexed sequents concerned by the rule $\square R$. All theses cases can be treated in an analogous way and in all of them the rule $\square R$ is clearly preserved. We show one of them ${ }^{2}$

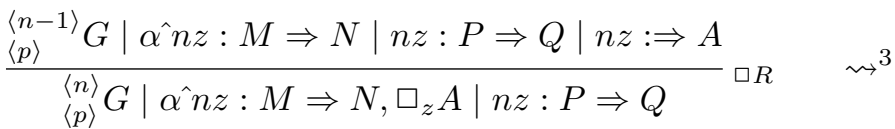

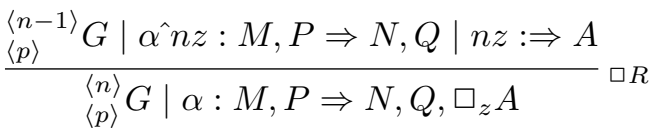

If the premise is inferred by the modal rule $\square L_{2}$, there are several cases to analyse depending on the indexes and the indexed sequents involved by

\footnotetext{
${ }^{2}$ We chose as example the case where the index $n z$ only occurs in the three displayed indexed sequents $M \Rightarrow N, P \Rightarrow Q, \Rightarrow A$.

${ }^{3}$ The symbol $\rightsquigarrow$ means: the premise of the right side is obtained by induction hypothesis on the premise of the left side.
} 
the rule of merge. All theses cases can be treated in an analogous way and in all of them the rule $\square L_{2}$ is clearly preserved. We show one of them ${ }^{4}$

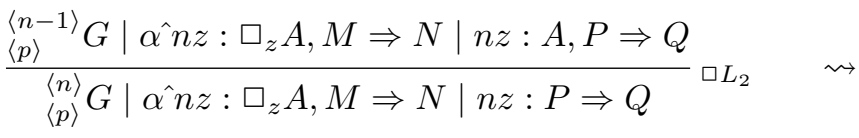

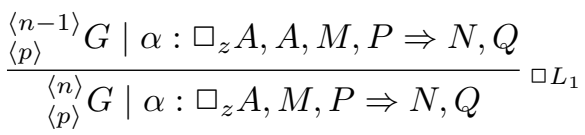

Lemma 4.6. The rule of external weakening:

$$
\frac{G \mid \alpha: M \Rightarrow N}{G|\beta: M \Rightarrow N| n z: P \Rightarrow Q} E W
$$

where $\beta=\left\{\begin{array}{ll}\alpha, & \text { if } n z \notin\|G\| \\ \alpha \hat{n} z, & \text { otherwise, }\end{array}\right.$ is height- and cut-rank preserving admissible in $\mathbf{H S 5}_{m}$.

Proof. By induction on the height of the derivation of the premise. If the premise is an initial indexed hypersequent, then so is the conclusion. If the premise is inferred by a propositional rule or by the rules $\square L_{1}$ and $\square L_{2}$, then the inference is clearly preserved. If the premise is inferred by the modal rule $\square R$, then all the cases can be treated by a straightforward induction, except the following one: ${ }^{5}$

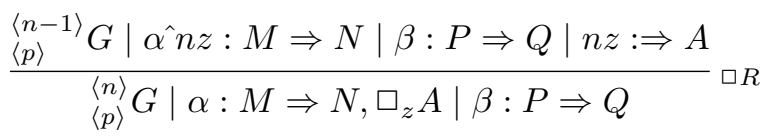

By applying Lemma 4.4 on the premise, we get ${ }_{\langle p\rangle}^{\langle n-1\rangle} G|\hat{\alpha} m z: M \Rightarrow N| \beta$ : $P \Rightarrow Q \mid m z: \Rightarrow A .^{6}$ We can now apply the inductive hypothesis obtaining ${ }_{\langle p\rangle}^{\langle n-1\rangle} G|\hat{\alpha} m z: M \Rightarrow N| \beta^{\wedge} n z: P \Rightarrow Q|m z: \Rightarrow A| n z: S \Rightarrow T$. Applying the rule $\square R$, we get the desired conclusion ${ }_{\langle p\rangle}^{\langle n\rangle} G\left|\alpha: M \Rightarrow N, \square_{z} A\right| \beta^{\wedge} n z$ : $P \Rightarrow Q \mid n z: S \Rightarrow T$.

\footnotetext{
${ }^{4}$ We chose as example the case where the index $n z$ only occurs in the two displayed indexed sequents $M \Rightarrow N$ and $P \Rightarrow Q$.

${ }^{5}$ We assume that the index $n z$ does not occur in $\|G\|$ nor in $\beta$.

${ }^{6}$ We assume that the index $m z$ does not occur in $\|G\|$ nor in $\beta$.
} 
Lemma 4.7. The rule of internal weakening:

$$
\frac{G \mid \alpha: M \Rightarrow N}{G \mid \alpha: M, P \Rightarrow N, Q}{ }^{I W}
$$

is height- and cut-rank-preserving admissible in $\mathbf{H S 5}_{m}$.

Proof. It follows from the height- and cut-rank-preserving admissibility of the two rules of merge and external weakening.

Lemma 4.8. The propositional rules of $\mathbf{H S 5}_{m}$ are height-and cut-rank-preserving invertible.

Proof. The proof proceeds by induction on the height of the derivation of the premise of the rule considered. The proof can be developed in the standard way. The only differences - the fact that we are dealing with indexed hypersequents and the cases where the last applied rule is one of the modal rules - are dealt with easily.

Lemma 4.9. The modal rules of $\mathbf{H S 5}_{m}$ are height- and cut-rank-preserving invertible.

Proof. The proof proceeds by induction on the height of the derivation of the premise of the rule considered. The rules $\square L_{i}$ are trivially height- and cutrank preserving invertible since both their premises are obtained by internal weakening from their respective conclusions, and weakening is height- and cut-rank preserving admissible.

We show in detail the invertibility of the rule $\square R$. We distinguish two main subcases: in one subcase $\beta=\alpha$, in the second subcase $\beta=\hat{\alpha} n z$. The two subcases can be treated in an analogous way, so we just show the proof of the former one in detail. If $G \mid \alpha: M \Rightarrow N, \square_{z} A$ is an initial indexed hypersequent, then so is $G|\hat{\alpha n z}: M \Rightarrow N| n z: \Rightarrow A$. If $G \mid \alpha: M \Rightarrow$ $N, \square_{z} A$ is obtained by a propositional rule $\mathcal{R}$, we first apply the inductive hypothesis on the premise(s), and then we apply the rule $\mathcal{R}$. If $G \mid \alpha: M \Rightarrow$ $N, \square_{z} A$ is of the form $G \mid \alpha: \square B, M^{\prime} \Rightarrow N, \square_{z} A$, then it may have been obtained by the two modal rules $\square L_{i}$. Since the procedure is the same for both rules, we just analyse the case of the rule $\square L_{1}$ and the other can be dealt with analogously. We apply the inductive hypothesis on $G \mid \alpha: \square B, B, M^{\prime} \Rightarrow$ $N, \square_{z} A$ and we obtain a derivation of height $n-1$ and the same cut-rank $p$ of $G\left|\hat{\alpha} n z: \square B, B, M^{\prime} \Rightarrow N\right| n z: \Rightarrow A$. By applying the rule $\square L_{1}$, we obtain a derivation of height $n$ and cut-rank $p$ of $G\left|\hat{\alpha} n z: \square B, M^{\prime} \Rightarrow N\right| n z: \Rightarrow A$. If $G \mid \alpha: M \Rightarrow N, \square_{z} A$ is obtained by the modal rule $\square R$ in which $\square_{z} A$ is not the principal formula, then this case can be treated analogously to the 
one of $\square L_{i}$. Finally, if $G \mid \alpha: M \Rightarrow N, \square_{z} A$ is obtained by the modal rule $\square R$ and $\square_{z} A$ is the principal formula, the premise of the last step gives the conclusion.

Lemma 4.10. The rules of contraction:

$$
\frac{G \mid \alpha: A, A, M \Rightarrow N}{G \mid \alpha: A, M \Rightarrow N} C L \quad \frac{G \mid \alpha: M \Rightarrow N, A, A}{G \mid \alpha: M \Rightarrow N, A} C R
$$

are height and cut-rank-preserving admissible in $\mathbf{H S} \mathbf{5}_{m}$.

Proof. By induction on the height of the derivation of the premise $G \mid \alpha$ : $M \Rightarrow N, A, A(G \mid \alpha: A, A, M \Rightarrow N)$. We only analyse the case of the rule $C R$. The case of the rule $C L$ can be treated similarly.

If $G \mid \alpha: M \Rightarrow N, A, A$ is an initial indexed hypersequent, so is $G \mid \alpha$ : $M \Rightarrow N, A$. If $G \mid \alpha: M \Rightarrow N, A, A$ is the conclusion of a rule $\mathcal{R}$ which does not have either of the two occurrences of the formula $A$ as principal, we apply the inductive hypothesis on the premise(s) and then we apply the rule $\mathcal{R}$. Suppose that $G \mid \alpha: M \Rightarrow N, A, A$ is the conclusion of a propositional or modal rule and one of the two occurrences of the formula $A$ is principal. Hence the last rule used in the derivation of $G \mid \alpha: M \Rightarrow N, A, A$ is a $R$-rule and we have to analyse the following three cases: $\neg R, \wedge R, \square R$.

$[\neg R]:$

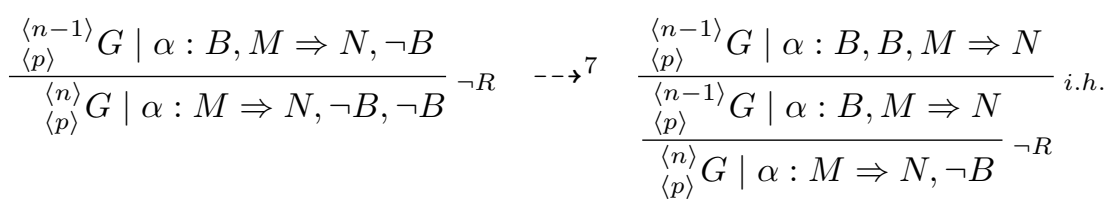

$[\wedge R]:$

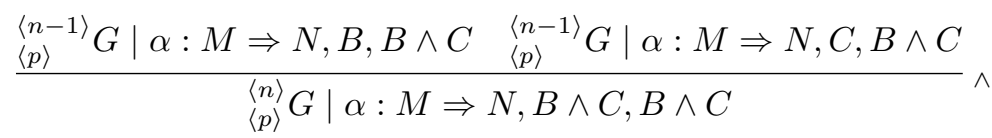

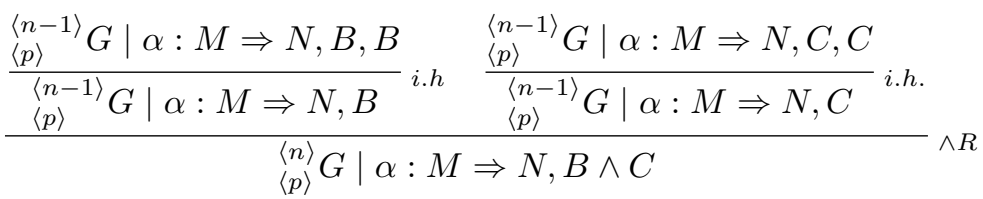

\footnotetext{
${ }^{7}$ The symbol $\rightarrow$ means: the premise of the right side is obtained by an application of Lemma 4.8 or Lemma 4.9 on the premise of the left side.
} 
$[\square R]:$

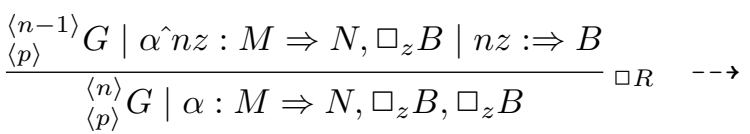

$$
\begin{aligned}
& \frac{\langle p\rangle}{\langle n-1\rangle} G|\hat{\alpha} n z: M \Rightarrow N| n z: \Rightarrow B \mid n z: \Rightarrow B_{\text {merge }}^{\langle\hat{\langle n n z}: M \Rightarrow N| n z: \Rightarrow B, B} \\
& \begin{array}{l}
\frac{\langle p\rangle}{\langle n-1\rangle} G|\hat{\alpha} n z: M \Rightarrow N| n z: \Rightarrow B, B^{\langle p\rangle} G|\hat{\alpha} n z: M \Rightarrow N| n z: \Rightarrow B
\end{array} \text { i.h. } \\
& { }_{\langle p\rangle}^{\langle n\rangle} G \mid \alpha: M \Rightarrow N, \square_{z} B \quad \square R
\end{aligned}
$$

Lemma 4.11. For all formulas $A$, we have $\mathbf{H S 5}_{m} \vdash_{0}^{2 \cdot d g(A)} G \mid \alpha: A, M \Rightarrow$ $N, A$.

Proof. By induction on the complexity of the formula $A$. The cases of atoms and propositional connectives are trivial. For $A=\square_{z} B$ we have

$$
\frac{G\left|\hat{\alpha n z}: \square_{z} B, M \Rightarrow N\right| n z: B \Rightarrow B}{\frac{G\left|\alpha \hat{n} z: \square_{z} B, M \Rightarrow N\right| n z: \Rightarrow B}{G \mid \beta: \square_{z} B, M \Rightarrow N, \square_{z} B} \square R}
$$

By induction we have a derivation of the premise of height $2 \cdot d g(B)$ and thus a derivation of the conclusion of height $2 \cdot d g(B)+2=2 \cdot(d g(B)+1)=$ $2 \cdot d g\left(\square_{z} B\right)$.

\section{The Adequateness Theorem}

In this section we show that the sequent calculus $\mathbf{H S 5}_{m}$ proves exactly the same formulas as its corresponding Hilbert-style system $\mathbf{S} \boldsymbol{5}_{m}$. (A detailed description of the Hilbert-style system $\mathbf{S} \mathbf{5}_{m}$ can be found in (5).)

Lemma 5.1. For all indexed hypersequent $G$, if $\vdash G$ in $\mathbf{H S 5}_{m}$, then $\vdash(G)^{\tau}$ in $\mathbf{S 5} \mathbf{5}_{m}$.

Proof. By induction on the height of derivations in $\mathbf{H S 5} \mathbf{5}_{m}$. As for the initial indexed hypersequents, the propositional rules and the rule $\square L_{1}$, the proof is straightforward. We analyse the case of the rule $\square L_{2}$. The case of the rule $\square R$ can be treated in an analogous way. Consider the rule $\square L_{2}$

$$
\frac{G\left|\hat{\alpha} n z: \square_{z} A, M \Rightarrow N\right| \hat{\beta} n z: A, P \Rightarrow Q}{G\left|\hat{\alpha} n z: \square_{z} A, M \Rightarrow N\right| \beta^{\wedge} n z: P \Rightarrow Q} \square L_{2}
$$


Let us denote with $H$ the indexed hypersequent occurring in the premise, and with $H^{\prime}$ the indexed hypersequent occurring in the conclusion. Suppose that $G$ is of the form $\alpha_{1}: \Gamma_{1}|\ldots| \alpha_{m-2}: \Gamma_{m-2}$. Then by the inductive hypothesis we have

$$
(H)_{\alpha_{1}: \Gamma_{1}}^{\tau} \wedge \ldots \wedge(H)_{\alpha_{m-2}: \Gamma_{m-2}}^{\tau} \wedge(H)_{\alpha^{\wedge} n z: \square_{z} A, M \Rightarrow N}^{\tau} \wedge(H)_{\beta^{\wedge} n z: A, P \Rightarrow Q}^{\tau}
$$

Consider $(H)_{\alpha_{r}: \Gamma_{r}}^{\tau}$, for $1 \leq r \leq m-2$, or $\alpha_{r}=\hat{\alpha} n z$ and $\Gamma_{r}=\square_{z} A, M \Rightarrow$ $N$. We have to show that if $\mathbf{S 5}_{m} \vdash(H)_{\alpha_{r}: \Gamma_{r}}^{\tau}$, then $\mathbf{S} 5_{m} \vdash\left(H^{\prime}\right)_{\alpha_{r}: \Gamma_{r}}^{\tau}$. This is trivial.

Consider $(H)_{\beta^{\wedge} n z: A, P \Rightarrow Q}^{\tau}$. We have to show that if $\mathbf{S 5}_{m} \vdash(H)_{\beta^{\wedge} n z: A, P \Rightarrow Q}^{\tau}$, then $\mathbf{S} 5_{m} \vdash\left(H^{\prime}\right)_{\beta^{\wedge} n z: P \Rightarrow Q}^{\tau}$. We proceed as follows, where each succeeding line follows forma the line above it by reasoning in $\mathbf{S} \mathbf{5}_{m}$.

$$
\begin{aligned}
& (A \wedge \wedge P \rightarrow \bigvee Q) \vee \square_{z}\left(\left(\square_{z} A \wedge \wedge M \rightarrow \bigvee N\right) \vee X\right) \vee Y \\
& \neg A \vee \neg(\bigwedge P) \vee \bigvee Q \vee \square_{z}\left(\neg \square_{z} A \vee \neg(\bigwedge M) \vee \vee N \vee X\right) \vee Y \\
& \square_{z} \neg \square_{z} A \vee \neg(\bigwedge P) \vee \vee Q \vee \square_{z}\left(\neg \square_{z} A \vee \neg(\bigwedge M) \vee \vee N \vee X\right) \vee Y \\
& \neg(\bigwedge P) \vee \vee Q \vee \square_{z}\left(\neg \square_{z} A \vee \neg \square_{z} A \vee \neg(\bigwedge M) \vee \vee N \vee X\right) \vee Y \\
& \neg(\bigwedge P) \vee \vee Q \vee \square_{z}\left(\neg \square_{z} A \vee \neg(\bigwedge M) \vee \bigvee N \vee X\right) \vee Y \\
& (\bigwedge P \rightarrow \bigvee Q) \vee \square_{z}\left(\left(\square_{z} A \wedge \wedge M \rightarrow \bigvee N\right) \vee X\right) \vee Y
\end{aligned}
$$

By the classical laws for conjunction, we have thus shown that

$$
\left(H^{\prime}\right)_{\alpha_{1}: \Gamma_{1}}^{\tau} \wedge \ldots \wedge\left(H^{\prime}\right)_{\alpha_{m-2}: \Gamma_{m-2}}^{\tau} \wedge\left(H^{\prime}\right)_{\alpha: \square_{z}}^{\tau} A, M \Rightarrow N \wedge\left(H^{\prime}\right)_{\beta^{\wedge} n z: P \Rightarrow Q}^{\tau}
$$

which is exactly what we were looking for.

Lemma 5.2. For all formulas $A$, if $\vdash A$ in $\mathbf{S 5} \mathbf{5}_{m}$, then $\vdash \Rightarrow$ in $\mathbf{H S 5} \mathbf{5}_{m}$.

Proof. By induction on the height of derivations in $\mathbf{H S} \mathbf{5}_{m}$. The classical axioms, the distribution axiom, the modus ponens and the rule of necessitation are proved as usual, we just present the proof of the axioms $T, 4, B$ and $5 .^{8}$

$\mathbf{H S 5}_{m} \vdash \square_{z} A \rightarrow A$

$$
\begin{aligned}
& \frac{\square_{z} A, A \Rightarrow A}{\square_{z} A \Rightarrow A} \\
& \Rightarrow \square_{z} A \rightarrow A
\end{aligned} \rightarrow R
$$

$\mathbf{H S 5}_{m} \vdash \Rightarrow \square_{z} A \rightarrow \square_{z} \square_{z} A$

\footnotetext{
${ }^{8}$ We are going to use the rule $\rightarrow R$, which can be derived by the logical rules of the calculus $\mathbf{H S 5}_{m}$, in order to simplify our rules.
} 


$$
\frac{1 z: \square_{z} A \Rightarrow|1 z: \Rightarrow| 1 z: A \Rightarrow A}{\frac{1 z: \square_{z} A \Rightarrow|1 z: \Rightarrow| 1 z: \Rightarrow A}{1 z: \square_{z} A \Rightarrow \mid 1 z: \Rightarrow \square_{z} A} \square R} \square\left[\begin{array}{l}
\square \\
\frac{\square_{z} A \Rightarrow \square_{z} \square_{z} A}{\Rightarrow \square_{z} A \rightarrow \square_{z} \square_{z} A} \rightarrow R
\end{array}\right.
$$

$\mathbf{H S 5}_{m} \vdash A \rightarrow \square_{z} \neg \square_{z} \neg A$

$$
\begin{gathered}
\frac{1 z: A \Rightarrow A \mid 1 z: \square_{z} \neg A \Rightarrow}{1 z: \neg A, A \Rightarrow \mid 1 z: \square_{z} \neg A \Rightarrow} \neg L \\
\frac{1 z: A \Rightarrow \mid 1 z: \square_{z} \neg A \Rightarrow}{1 z: A \Rightarrow \mid 1 z: \Rightarrow \neg \square_{z} \neg A} \neg R \\
\frac{A L_{2}}{\Rightarrow \Rightarrow \square_{z} \neg \square_{z} \neg A} \\
\frac{A}{\Rightarrow A \rightarrow \square_{z} \neg \square_{z} \neg A} \rightarrow R
\end{gathered}
$$

$\mathbf{H S F}_{m} \vdash \Rightarrow \square_{z} \neg A \rightarrow \square_{z} \neg \square_{z} \neg A$

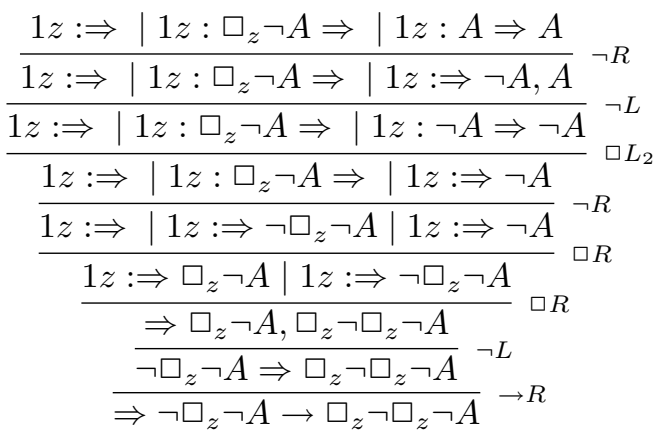

Corollary 5.3. The calculus $\mathbf{H S 5}_{m}$ is sound and complete with respect to the Hilbert system $\mathbf{S 5}_{m}$.

Proof. Follows from Lemmas 5.1 and 5.2.

\section{Cut-elimination Theorem}

In this section we prove that the cut-rule is eliminable in the calculus $\mathbf{H S F}_{m}$, and that the cut-elimination procedure yields an upper bound of $h p_{2}(n, 0)$ where $h p_{2}$ is a hyperexponential function at base 2 defined as follows. 
Definition 6.1. The hyperexponential function of base $2, h p_{2}: \mathbb{N}^{2} \rightarrow \mathbb{N}$, is recursively defined in the following way:

$h p_{2}(m, 0)=m$

$h p_{2}(m, k+1)=2^{h p_{2}(m, k)}$

We write $2_{k}(m)$ instead of $h p_{2}(m, k)$. Hence:

$2_{0}(m)=m$

$2_{k+1}(m)=2^{2_{k}(m)}$

In what follows, given a derivation $d$, we denote its height by $|d|$.

Lemma 6.2. (Reduction Lemma) Given two derivations

$$
d_{1} \vdash_{p}^{\left|d_{1}\right|} G\left|\alpha: M \Rightarrow N, A \quad d_{2} \vdash_{p}^{\left|d_{2}\right|} G\right| \alpha: A, M \Rightarrow N
$$

with $d g(A)=p$, we can effectively construct a derivation

$$
d \vdash_{p}^{\left|d_{1}\right|+\left|d_{2}\right|} G \mid \alpha: M \Rightarrow N
$$

Proof. By induction on $\left|d_{1}\right|+\left|d_{2}\right|$. We perform a case analysis on the two lowermost rules in the given proofs. Case 1 . If one of the two rules is passive and an axiom, then $G \mid \alpha: M \Rightarrow N$ is axiomatic as well. Case 2. If one is active and an axiom, then we have

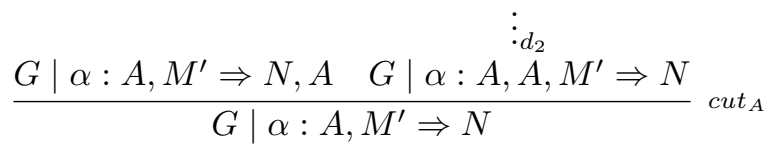

which reduces to

$$
\frac{\stackrel{\vdots}{d_{2}}}{G \mid \alpha: A, A, M^{\prime} \Rightarrow N} \underset{ }{G \mid \alpha: A, M^{\prime} \Rightarrow N} C L
$$

By Lemma 4.10 we have $\vdash_{p}^{\left|d_{2}\right|} G \mid \alpha: A, M^{\prime} \Rightarrow N$, and hence $\vdash_{p}^{\left|d_{1}\right|+\left|d_{2}\right|} G \mid \alpha$ : $A, M \Rightarrow N$.

Case 3 . If some rule $\mathcal{R}$ is passive, then we have

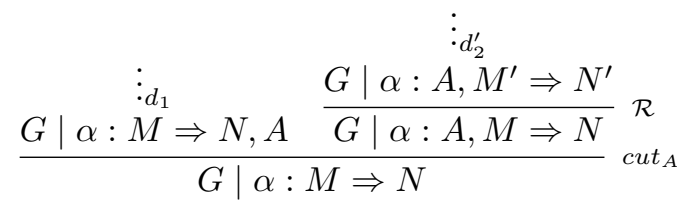

we reduce to 


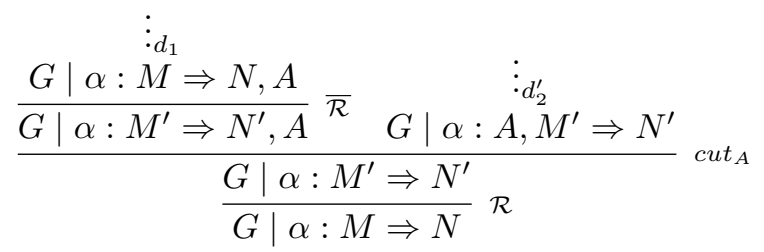

By Lemmas 4.8 or 4.9 , we get $\vdash_{p}^{\left|d_{1}\right|} G \mid \alpha: M^{\prime} \Rightarrow N^{\prime}, A$. Thus, by inductive hypothesis, $\vdash_{p}^{\left|d_{1}\right|+\left|d_{2}^{\prime}\right|} G \mid \alpha: M^{\prime} \Rightarrow N^{\prime}$, and by the rule $\mathcal{R}$ we get $\vdash_{p}^{\left|d_{1}\right|+\left|d_{2}\right|}$ $G \mid \alpha: M \Rightarrow N$.

Note that we have only analysed the case where $\mathcal{R}$ is a one-premise rule and it operates on the sequent $\alpha: A, M \Rightarrow N$. The cases where $\mathcal{R}$ is a two-premise rules or it operates on $G$ can be treated in a similar way.

Case 4 . We are left with the case where both rules are active and no axioms. We have:

$$
\begin{aligned}
& (\neg R-\neg L \text { rules }) \text { : } \\
& { }_{d_{1}^{\prime}} \\
& \frac{\frac{G \mid \alpha: B, M \Rightarrow N}{G \mid \alpha: M \Rightarrow N, \neg B} \neg R \quad \frac{G \mid \alpha: M \Rightarrow N, B}{G \mid \alpha: \neg B, M \Rightarrow N} \neg L}{G \mid \alpha: M \Rightarrow N} c u t_{\neg B}
\end{aligned}
$$

we reduce to

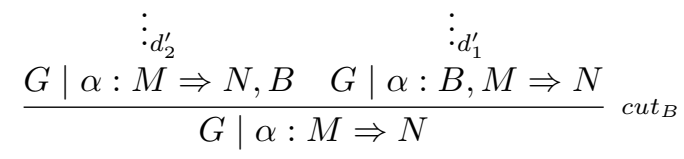

where $\vdash_{p}^{\left|d_{1}\right|+\left|d_{2}\right|} G \mid \alpha: M \Rightarrow N$ since $\max \left(\left|d_{1}^{\prime}\right|,\left|d_{2}^{\prime}\right|\right)+1 \leq\left|d_{1}\right|+\left|d_{2}\right|$, and $d g(B)<d g(\neg B)=p$.

$$
\begin{aligned}
& (\wedge R-\wedge L \text { rules }):
\end{aligned}
$$

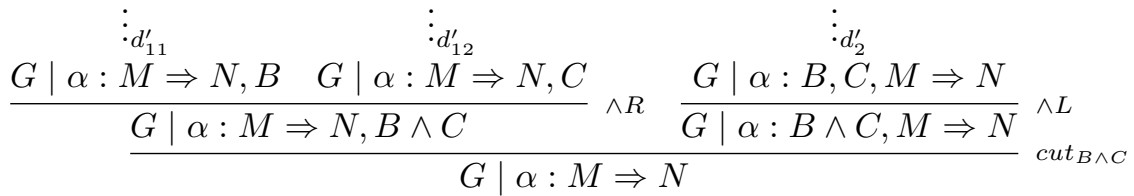

we reduce to: 


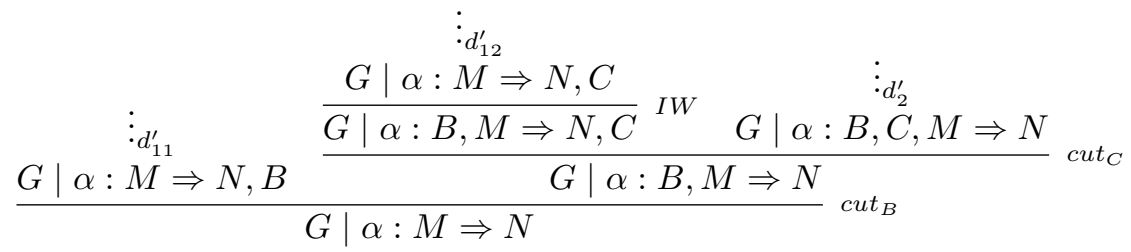

By Lemma 4.7 we get $\vdash_{p}^{\left|d_{12}^{\prime}\right|} G \mid \alpha: B, M \Rightarrow N, C$, and since $d g(C)$, $d g(B),<d g(B \wedge C)=p$, we get $\vdash_{p}^{r} G \mid \alpha: M \Rightarrow N$, where $r=\max \left(\left|d_{11}^{\prime}\right|, \max \left(\left|d_{12}^{\prime}\right|,\left|d_{2}^{\prime}\right|\right)+\right.$ $1)+1$. It is easy to check that $r \leq\left|d_{1}\right|+\left|d_{2}\right|$

$\left(\square R-\square L_{1}\right.$ rules):

$$
\begin{aligned}
& \vdots_{d_{1}^{\prime}} \vdots_{d_{2}^{\prime}}
\end{aligned}
$$

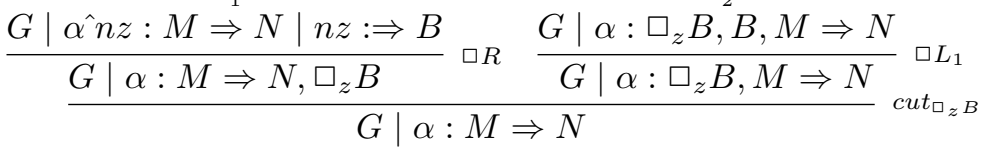

we reduce to

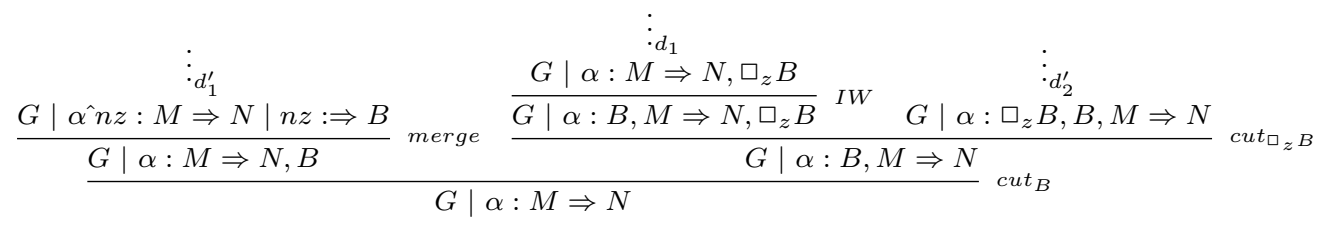

where the left premise of the upper cut has been derived by the use of Lemma 4.7 with height $\left|d_{1}^{\prime}\right|+1$ and $\left|d_{2}^{\prime}\right|$, the sum of which is smaller than $\left|d_{1}\right|+\left|d_{2}\right|$. The inductive hypothesis thus yields $\vdash_{p}^{\left(\left|d_{1}^{\prime}\right|+1\right)+\left|d_{2}^{\prime}\right|} G \mid \alpha: B, M \Rightarrow N$, and since $d g(B)<d g\left(\square_{z} B\right)=p$ and the left premise has been obtained by Lemma 4.5 , we get $\vdash_{p}^{\left|d_{1}\right|+\left|d_{2}\right|} G \mid \alpha: M \Rightarrow N$, by the lower cut.

Note that we have analysed the case where the index $n z$ only appears in the displayed sequents $M \Rightarrow N$ and $\Rightarrow B$. The case where $n z \in\|G\|$ can be dealt with analogously.

$$
\left(\square R-\square L_{2}\right. \text { rules): }
$$




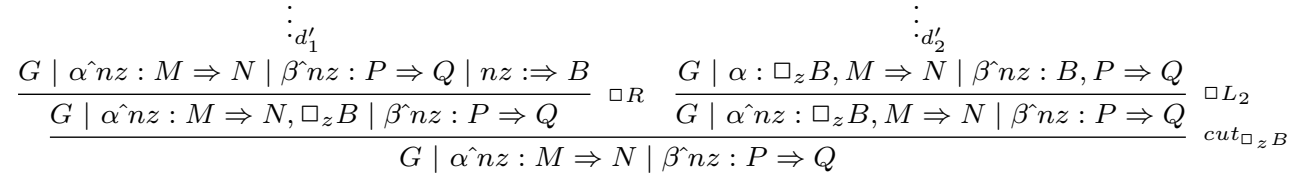

we reduce to

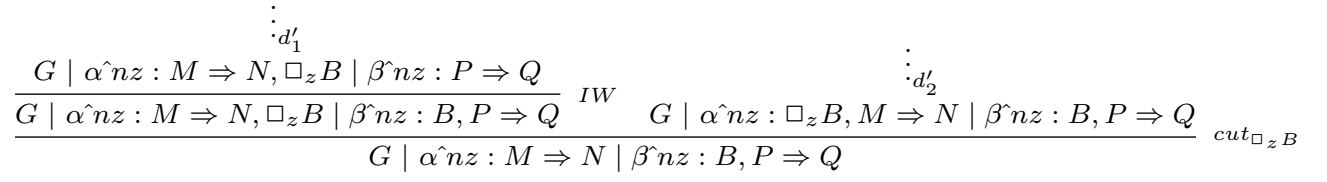

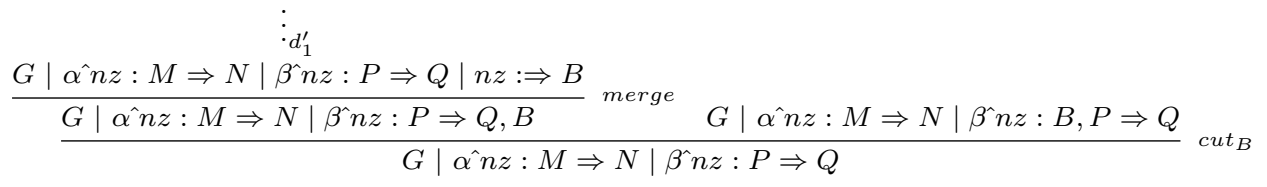

where the left premise of the upper cut has been derived by the use of Lemma 4.7 with height $\left|d_{1}^{\prime}\right|+1$ and $\left|d_{2}^{\prime}\right|$, the sum of which is smaller than $\left|d_{1}\right|+\left|d_{2}\right|$. The inductive hypothesis thus yields $\vdash_{p}^{\left(\left|d_{1}^{\prime}\right|+1\right)+\left|d_{2}^{\prime}\right|} G|\hat{\alpha \hat{n}} n: M \Rightarrow N| \beta^{\wedge} n z$ : $B, P \Rightarrow Q$, and since $d g(B)<d g\left(\square_{z} B\right)=p$ and the left premise has been obtained by Lemma 4.5, we get $\vdash_{p}^{\left|d_{1}\right|+\left|d_{2}\right|} G|\hat{\alpha} n z: M \Rightarrow N| \beta^{\wedge} n z: P \Rightarrow Q$, by the lower cut.

From the reduction lemma we obtain the first and the second elimination lemma as usual, e.g. see (8) or (9).

Lemma 6.3. (First Elimination Lemma) If $\vdash_{p+1}^{m} G$, then $\vdash_{p}^{2^{m}} G$.

Lemma 6.4. (Second Elimination Lemma) If $\vdash_{p}^{n} G$, then $\vdash_{0}^{2_{p}(n)} G$.

Lemma 6.5. (Cut Elimination) If $A$ is a valid formula, then $\vdash_{0}^{2_{p}(n)} \Rightarrow A$.

\section{Decidability}

In this section we prove that the calculus $\mathbf{H S 5 _ { m }}$ is decidable, i.e. there is an algorithm that, given any indexed hypersequent $G$, determines whether $G$ is provable in $\mathbf{H S} \boldsymbol{5}_{m}$ or not.

First of all let us observe that our calculus satisfies the subformula property since: (i) the cut rule is eliminable in it, and (ii) in each of its 
rules all the formulas which occur in the premise(s) are subformulas of the formulas which occur in the conclusion. Moreover, it can also be shown that the contraction rules are admissible (see Lemma 4.10). It would therefore seem that any source of potentially non-terminating proof search had been cut off. Unfortunately it is not so because of the repetition of the principal formula in each of the rules $\square L_{i}$. In order to avoid this problem and prove that our calculus is decidable, we shall obtain a bound on the number of applications of the rules $\square L_{i}$.

For this goal, let us start by only taking into account minimal derivations, which is to say, derivations where shortenings are not possible. Then we prove, by means of the following lemmas and their corollaries, that in minimal derivations it is enough to apply the rule $\square L_{1}$ only once on any given pair of principal formulas and the rule $\square L_{2}$ only once on any given pair of sequents. This technique is analogous to that used in (7).

Lemma 7.1. The rule $\square L_{1}$ permutes down with respect to the other logical (propositional and modal) rules.

Proof. The cases of permutation with a one-premise propositional rule and with the modal rules are straightforward. Let us consider the permutation with the two premises-rule $\wedge R$. We have the following derivation

$$
\begin{aligned}
& \frac{\frac{G \mid \alpha: \square_{z} A, A, M \Rightarrow N, B}{G \mid \alpha: \square_{z} A, M \Rightarrow N, B} \square L_{1} \quad G \mid \alpha: \square_{z} A, M \Rightarrow N, C}{G \mid \alpha: \square_{z} A, M \Rightarrow N, B \wedge C} \wedge R \\
& \downarrow
\end{aligned}
$$

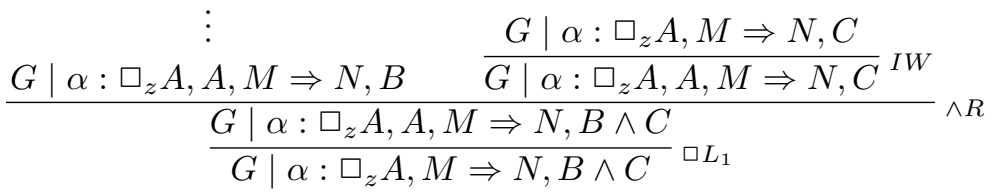

Let us remark that the transformation of the first derivation into the second one is done by means of an application of the height- and cut-rank-preserving admissible rule of internal weakening.

Lemma 7.2. The rule $\square L_{2}$ permutes down with respect to the propositional rules and the rule $\square L_{1}$. It also permutes with the instances of $\square R$ where the principal formula $A$ of the premise of $\square L_{2}$ is not active in the sequent where the principal formula of the premise of $\square R$ occurs. 
Proof. The cases of permutation with the propositional rules and the rule $\square L_{1}$ can be dealt with analogously to the corresponding cases of the previous lemma. We show the permutation in case of the rule $\square R$. We underline that this case is constrained by the hypothesis that the principal formula $A$ of the premise of the rule $\square L_{2}$ is not active in the same sequent where the principal formula of the premise of the rule $\square R$ occurs. By taking into account this restriction, the permutation is straightforward:

$$
\begin{aligned}
& \frac{G\left|\hat{\alpha \wedge} z: \square_{z} A, M \Rightarrow N\right| \beta^{\wedge} n z: A, P \Rightarrow Q \mid n z: \Rightarrow B}{G\left|\hat{\alpha} n z: \square_{z} A, M \Rightarrow N\right| \beta^{\wedge} n z: P \Rightarrow Q \mid n z: \Rightarrow B} \square L_{2} \\
& \frac{G\left|\hat{\alpha} n z: \square_{z} A, M \Rightarrow N,\right| \hat{\beta} n z: A, P \Rightarrow Q \mid n z: \Rightarrow B}{G\left|\hat{\alpha n z}: \square_{z} A, M \Rightarrow N, \square_{z} B\right| \beta^{\wedge} n z: A, P \Rightarrow Q} \underset{\square}{G\left|\hat{\alpha} n z: \square_{z} A, M \Rightarrow N, \square_{z} B\right| \beta^{\wedge} n z: P \Rightarrow Q}
\end{aligned}
$$

The cases that differ from this one just from the point of view of indexes can be treated in a similar way.

Corollary 7.3. In a minimal derivation in $\mathbf{H S 5}_{m}$, the rule $\square L_{1}$ cannot be applied more than once on the same pair of principal formulas of any branch.

Proof. Suppose we have a minimal derivation where the rule $\square L_{1}$ has been applied twice on the same pair of sequents:

$$
\begin{gathered}
\frac{G^{\prime} \mid \alpha: \square_{z} A, A, M^{\prime} \Rightarrow N^{\prime}}{G^{\prime} \mid \alpha: \square_{z} A, M^{\prime} \Rightarrow N^{\prime}} \square L_{1} \\
\cdot \\
\cdot \\
\cdot \\
\frac{G \mid \alpha: \square_{z} A, A, M \Rightarrow N}{G \mid \alpha: \square_{z} A, M \Rightarrow N} \square L_{1}
\end{gathered}
$$

By permuting the rule $\square L_{1}$ down with respect to the the steps in the dotted part of the derivation, we obtain a derivation of the same height ending with: 


$$
\frac{G \mid \alpha: \square_{z} A, A, A, M \Rightarrow N}{\frac{G \mid \alpha: \square_{z} A, A, M \Rightarrow N}{G \mid \alpha: \square_{z} A, M \Rightarrow N} \square L_{1}}
$$

By applying the height- and cut-rank-preserving admissible rule $C L$ on the two occurrences of the formula $A$ in place of the upper $\square L_{1}$, we obtain a shorter derivation, contrary to the assumption of minimality.

Corollary 7.4. In a minimal derivation in $\mathbf{H S 5}_{m}$, the rule $\square L_{2}$ cannot be applied more than once on the same pair of sequents of any branch.

Proof. Consider a minimal derivation where the rule $\square L_{2}$ has been applied twice on the same pair of sequents:

$$
\begin{gathered}
\frac{G^{\prime}\left|\hat{\alpha} n z: \square_{z} A, M^{\prime} \Rightarrow N^{\prime}\right| \beta^{\wedge} n z: A, P^{\prime} \Rightarrow Q^{\prime}}{G^{\prime}\left|\hat{\alpha} n z: \square_{z} A, M^{\prime} \Rightarrow N^{\prime}\right| \beta^{\wedge} n z: P^{\prime} \Rightarrow Q^{\prime}} \\
\cdot \\
\cdot \\
\cdot \\
\frac{G\left|\hat{\alpha} n z: \square_{z} A, M \Rightarrow N\right| \beta^{\wedge} n z: A, P \Rightarrow Q}{G\left|\hat{\alpha} n z: \square_{z} A, M \Rightarrow N\right| \beta^{\wedge} n z: P \Rightarrow Q} \square L_{2}
\end{gathered}
$$

By permuting down $\square L_{2}$ with respect to the steps in the dotted part of the derivation, we obtain a derivation of the same height ending with:

$$
\frac{G\left|\hat{\alpha} n z: \square_{z} A, M \Rightarrow N\right| \beta^{\wedge} n z: A, A, P \Rightarrow Q}{G\left|\hat{\alpha} n z: \square_{z} A, M \Rightarrow N\right| \beta^{\wedge} n z: A, P \Rightarrow Q} \square \square L_{2}
$$

By applying the height- and cut-rank-preserving admissible rule $C L$ on the two occurrences of the formula $A$ in place of the upper $\square L_{2}$, we obtain a shorter derivation, contrary to the assumption of minimality.

Finally we underline that if the principal formulas of the premise of the upper application of the rule $\square L_{2}$ were active in the sequent where the principal formula of the premise of $\square R$ occurs, then that sequent would disappear and therefore we would not find it in the premise of the lower application of the rule $\square L_{2}$. The conclusion is that the restriction of Lemma 7.2 is not limitative for the proof of this corollary. 
Now we prove that the modal logic $\mathbf{S} \boldsymbol{5}_{m}$ is decidable by showing effective bounds on proof search in the calculus $\mathbf{H S 5}_{m}$.

Theorem 7.5. The calculus $\mathbf{H S 5}_{m}$ allows terminating proof search.

Proof. Place an indexed hypersequent $G$, for which we are looking for a proof search, at the root of the procedure. Apply first the propositional rules and then the modal rules. The propositional rules reduce the complexity of the indexed hypersequent. The rule $\square R$ removes the modal constant $\square_{z}$ and adds a new sequent, each of the rules $\square L_{i}$ increases the complexity. However, by the corollary 7.3 , the rule $\square L_{1}$ cannot be applied more than once on the same pair of principal formulas, while, by the corollary 7.4 , the rule $\square L_{2}$ cannot be applied more than once on the same pair of sequents. Therefore the number of applications of the two rules $\square L_{1}$ and $\square L_{2}$ is bounded, respectively, by the number of $\square_{z}$ 's occurring in the negative part (see definition below) of the indexed hypersequent to prove, and by the number of the indexed sequents which may appear in the derivation. This latter, in its turn, is bounded by the number of indexed sequents belonging to the hypersequent to prove, and the indexed sequents which can be introduced by applications of the rule $\square R$.

We finally explain how to calculate explicit bounds on the number of applications of the rules $\square L_{i}$. First of all, define the negative and positive parts of the hypersequent $\alpha_{1}: M_{1} \Rightarrow N_{1}|\ldots| \alpha_{n}: M_{n} \Rightarrow N_{n}$, as the negative and positive parts of each of the following conjuncts and disjuncts:

$$
\bigwedge M_{1} \rightarrow \bigvee N_{1}, \ldots, \bigwedge M_{n} \rightarrow \bigvee N_{n}
$$

For any given indexed hypersequent $G$, let $n\left(\square_{z}\right)$ be the number of $\square_{z}$ 's in the negative part of the indexed hypersequent $G$, and $p\left(\square_{z}\right)$ be the number of $\square_{z}$ 's in the positive part of the indexed hypersequent $G$. The number of applications of the rule $\square L_{1}$ in a minimal derivation is bounded by $n\left(\square_{z}\right)$.

In the case where the root-indexed-hypersequent is just an indexed sequent, the number of applications of the rule $\square L_{2}$ in a minimal derivation is bounded by $n(\square) \cdot p(\square)$. In the case where the root-indexed-hypersequent is an indexed hypersequent and $n$ is the number of indexed sequents which occurs in it, the number of applications of the rule $\square L_{2}$ in a minimal derivation is bounded by: $n(\square) \cdot(p(\square)+n)$.

\section{References}

[1] R. J. Aumann, Agreeing to disagree. Annals of Statistics 4 (1976), 12361239.

[2] P. Balbiani, and H. van Ditmarsch, and A. Herzig, and T. de Lima, Tableaux for public announcements. Journal of Logic and Computation 20 (2010), 55-76. 
[3] A. Baltag, and L. Moss, Logic for epistemic programs. Synthese $\mathbf{1 3 9}$ (2004), 165-224.

[4] C. Cerrato, Cut-free Modal sequents for normal modal logics. NotreDame Journal of Formal Logic 34 (1993), 564-582.

[5] H. Van Ditmarsch, and W. Van der Hoek, and B. Kooi, Dynamic Epistemic Logic. Synthese, 2007.

[6] A. Indrzejczak, Cut-free Double Sequent Calculus for S5. Logic Journal of the IGPL 4 (1998), 505-516.

[7] F. Poggiolesi, A cut-free simple sequent calculus for modal logic S5. Review of Symbolic Logic, 1 (2008), 3-1-5.

[8] W. Pohlers, Proof Theory - An introduction. Springer, 1989.

[9] K. Schütte, Proof Theory. Springer, 1977

[10] A. Troelestra, and H. Schwichtenberg, Basic Proof Theory. Cambridge University Press, 1996.

[11] H. Wansing, Sequent Calculi for Normal Modal Propositional Logics. Journal of Logic and Computation 4 (1998), 125-142.

Francesca Poggiolesi 